\title{
Business Intelligence or Intelligent Business?
}

\author{
Gustavo A. Ortiz \\ Universidad Nacional de Asunción \\ *Corresponding Author: gustavo.ortizd@gmail.com
}

Copyright $(2014$ Horizon Research Publishing All rights reserved.

\begin{abstract}
The implementation of Business Intelligence (BI) in companies requires a high degree of commitment from top level management and directors, a change in culture and organizational maturity, a true Metanoia. It is not only a matter of efficiently incorporate a new technology platform; it is an integrated new business. If given these and other conditions, the result will be a smarter and more competitive business.
\end{abstract}

Keywords BI, IT, Innovation, Business Intelligence, Metanoia, ERP, ICT

\section{Introduction}

This work explains the main concepts related to Business Intelligent and its implementation in companies as well as understand the causes of its failures and how to avoid them and principally, what should companies do?

Before entering the BI specific matter, it is important to remember that data analysis is a process of inspecting, cleaning and transforming data with the goal of highlighting useful information, suggesting conclusions, and supporting decision making. Data analysis has multiple facets and approaches, encompassing diverse techniques in a variety of names, in different business, science, and social science domains.

\subsection{Business Intelligence}

Business Intelligence (BI) is an expression with which, especially in the field of computer service offerings, refers to the scope of organizations aimed at promoting greater efficiency in decision making. Implementing Business Intelligence in the company, depending on the context and objectives involves innovation at least in processes. In his book "The Future of Management" Gary Hamel argues that organizations need management innovation now more than ever. What is the reason for this statement? The management paradigm of the last century, focusing on the control and efficiency is not enough, we live in a world where adaptability and creativity (freeing the mind of the polarizing effect of old ideas), are key to the success of business.
To thrive into the future, companies must reinvent management and also, constantly reinvent themselves, since at the moment that the company slows, another one takes advantage and given the impressive technological development that can lead to be unrecoverable, and then being relegated to oblivion, with the label of "displaced by another more competitive and intelligent company."

\section{Methodology}

In order to accomplish this investigation, several sources of information were used. A literature search was performed in September 2010 at the Universidad Nacional de Asunción library, and also its digital library and materials from Universidad de Buenos Aires during the MBA course in Asuncion that year. Besides this sources, were used Internet and a survey to 10 selected Paraguayan IT companies that usually implement IT solutions in commercial and industrial organizations, mainly SMEs (Small and Medium Enterprises).

\subsection{Metanoia (Business Transformation) and Systemic Thinking}

There are many companies who are facing a rapid decline of revenues and market bleak prospects, those that fit with a more proactive approach to "do more with what they have "come out of the current crisis as clear winners. Though cuts in capital expenditure budgets have slowed many BI initiatives, there is much that can be done to obtain a significant strategic and operational improvement while implementing BI properly.

\subsection{But What Should the Organization Effectively Do?}

According to Peter Senge, metanoia, means a change of approach, refers to systemic thinking, the fifth discipline of learning organizations, to see "the whole elephant" and not just fractions of it and each with a different interpretation, where the sum of the parts, paradoxically is not equal to the total. This would be the key aspect, not only as the basis for the successful implementation of BI, but necessary for the survival of the company.

"The idea of the learning organization is a mental 
revolution. It requires us to abandon old paradigms of leadership and power to approach a thought capable of dealing with complex systems. No one would ever teach a child writing hand training alone. But we still believe that for a company sells, achieved with a good team of vendors and a lot of publicity."

Systems thinking is also related to the theory of systems. "The development of systems theory gave rise to a new way of seeing the world. This systems approach appeared as a reaction to the excessive generated super specialist factoring sciences early twentieth century. At that time, it was noted that required science scholars who, knowing little of the details, saw the whole. Scientists realized that, due to the interaction between the parties, assumes all attributes that would lack if eliminated or modified some relationships.

The systems approach involves having a concept while analyzing all parts, like a puzzle. It is an integrated thinking, even when the analysis is done part by part. Allows a better understanding of the nature of the problems and decrease the difficulty of analysis. (Saroka-Collazo, 2010)

Business Intelligence is well known for its ability to integrate, to optimize both the cost and revenue activities of an organization. What is less known is the power of $\mathrm{BI}$ as a transformation tool. Using Business Intelligence approach driven transformation programs of the company, companies can dramatically increase their chances of adding value to the business.

The technology implementation failures Despite the theoretical assumptions above, however, contrasts sharply with reality, there are numerous failures to implement ICT organizations obviously BI is within the ICT or IT world. According Piorun (2010), this is supported by several surveys that say that only in the order of $20 \%$ of projects end getting the stated objective, in time, with the estimated resources. This problem occurs in all types of projects, and is particularly found in technology projects. In these, $80 \%$ of the projects were not so lucky - for different reasons - leads to higher direct costs (in some cases, larger projects are completed with much more resources than expected) and finally, not reaching the expected benefits that would provide if the project it had completed in a timely manner.

In the final analysis, in addition to the direct costs that are easily countable, the indirect costs are probably much more important than what may be expected, if not, probably companies are promoting projects that did not add much value to the organization. This fundamentally impacts in low productivity of some areas of the organization and in the cost of opportunity of capital that is invested in the initiative.

\subsection{Some Causes}

As mentioned above and the findings of Piorun (2010), at this point, it is important to analyze the causes of failures technology. The research of Markowsky in 2004, shows that in a large percentage (92\%), the causes of failure in IT project implementations point to organizational factors, cultural and human, while only $4 \%$ is attributed to technological factors. This further strengthens the hypothesis of this study that organizations should be smart. This investigation that took place in 2013 , found that $90 \%$ of projects exceed the initial budget and the reason was not the increase in the scope of the project but a incomplete prospecting of system needs. See Figure 1.

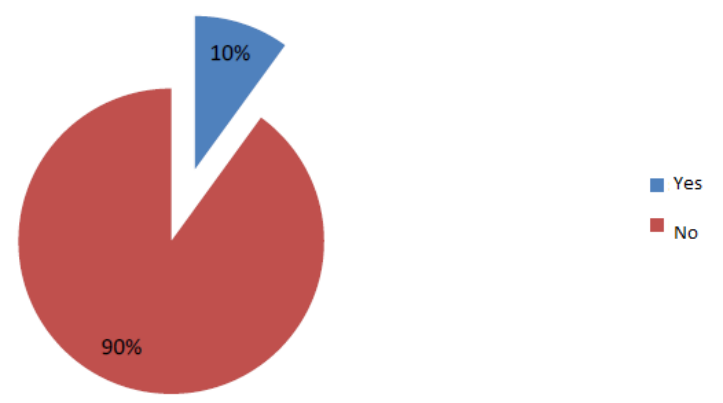

Figure 1. Showing most projects in Paraguayan SMEs exceed initial budget.

Another finding of this work is the fact that a high percentage $(80 \%)$ of selected SMEs owners had active participation from purchase to implementation of these information systems and those who for various reasons have been unable to follow the process indicated that for just that reason systems were not successful or not be implemented properly. In a similar proportion, owners participated closely with senior management in the process of purchasing and implementing systems, this being a very favorable combination for success. See Figure 2.

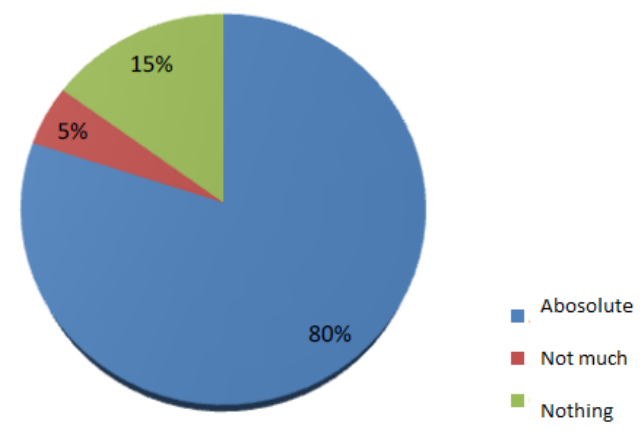

Figure 2. showing how relevant was owners participation in success cases.

\subsection{A transformer BI company}

In a much more optimistic approach, organizational transformations due to $\mathrm{BI}$ occur at several levels:

1. BI ensures that all processes are directly linked to the strategic objectives. BI efforts, like many other IT initiatives cannot be delegated to the CIO, it is a business project with the aid of technology. Companies should form a team of people involved and, most important, committed to the project.

2. The embedded information ensures that the processes make intelligent decisions based on data.

3. Automated alerts and decision can significantly 
accelerate the delivery cycle.

4. Process monitoring and feedback through dashboards ensure continuous improvement.

This extends the value of BI beyond the ability to extract, aggregate and analyze data. Using BI allows significantly change technology and how a company responds to its organizational design challenges.

\subsection{BI and Enterprise}

Most organizational development initiatives are driven by the costs and revenue optimization objectives, which support the concept that to be more agile, more efficient and more competitive, the organization needs to improve its strategic value chains, rather than functional business areas such as Finance, Marketing, Logistics, HR, etc..

With the large volume of data generated by businesses today, the integration of BI technologies in Enterprise Resource Planning (ERP) and Enterprise Data Warehouse (EDW), allows these data to release, effectively, intelligence on internal operations and outside the company.

The implementation of a Business Intelligence system requires you undertake a project including consultancy services for design and implementation of mechanisms for data conversion and implementation. Technology is just a tool that must be properly exploited and should be selected with attributes of scalability, support and integration of products from different suppliers.

\subsection{Talking about Data Warehouse}

The installation of a Data Warehouse is just the beginning of the road to get to provide users with the right tools and information they need. According W. H. Immon, creator of the concept, a Data Warehouse is "a set of integrated data, subject-oriented, time-varying and non-transient, which support the decision making process of an administration."

Therefore, its success requires that begins with the clear identification of business requirements, then, on the basis thereof, shall be determined by the design of the Data Warehouse and applicable tools for analysis, such as advanced interfaces, Data mining and OLAP systems based on Web formats.

\subsection{Features Data Warehouse}

After reviewing the literature of the specific issue, features can be summarized as follows:

- Organized around themes. Information is based on the aspects that are of interest to the company.

- Integrated. It is the most important aspect. Data integration involves naming conventions, coding consistent, uniform measure variables, etc.

- Time dependent. This dependence appears in three forms:

- The information represents the data over a long time horizon.
- $\quad$ Each key structure contains (implicitly or explicitly) an element of time (day, week, month, etc.).

- The information, once properly registered, cannot be updated.

- $\quad$ Non-volatile. The Data Repository allows uploading new data and access the already stored, but does not allow delete or modify data

\subsection{Logical Structure}

The logical structure of a data warehouse consists of the following levels:

- Metadata: describe the structure of the data in the warehouse.

- They are in a different dimension to the other levels.

- Detailed data current.

- Obtained directly from data processing.

- They are the lowest level of detail.

- They take up much space.

- Is stored on disk, for easy access.

- Detailed data history.

- Same as above, but with data from the past.

- Is usually stored on an external medium, because access is rare.

- Lightly summarized data.

- First level of aggregation of the current detailed data.

- Correspond to common queries.

- Is stored on disk.

- Highly summarized data. Are the highest level of aggregation.

- Correspond to queries made very often and to be obtained very quickly.

- They are usually separated from the data store, forming Data Supermarkets (Data Marts).

Figure 3 shows the Data Warehouse, embedded in a Business Intelligence system.

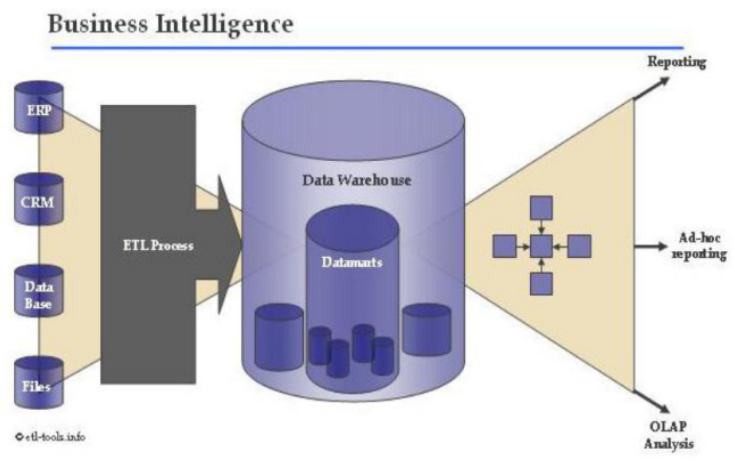

Figure 3. A typical Business Intelligence system

The term ETL means Extract, Transform and Load data (for its acronym in English). As an alternative it may be proposed data transfer first, and then do all the transformations in the destination. In simple terms what is ETL? They are all necessary activities related to the management of data and metadata in order to meet the information needs. The terms ERP, CRM will be discussed 
in detail later.

Imagine our plan of BI as a large clock, where ETL processes are represented by many small and large gears to make sure everything works as planned accurately and efficiently. It is important to note that any damage propagates the error in the system.

ETL architecture should be considered as a separate service from query and data presentation, it is hardware and software used to implement this by following the best practices of each vendor and those that indicate experience.

The ETL process, as we see, is not a minor issue. From a technological point of view is a KSF (Key Success Factor) of a BI solution, to automate and simplify complex or time demanding processes, allowing storing and handling all of the solutions. Most Data Warehouse projects incorporate ETL processes, it is common in over $80 \%$ of these projects an item for ETL, but not in operational systems, where code or the application itself are still used for data movement.

The ETL process is not perceived from the point of view of the end user information systems (except certain data mining or analysis), however, users have an important role in the design and development of these processes.

It is the business that raises their information needs and initial system requirements.

This raises two important requirements on the data that must be met by the ETL process:

1) The information needs determine the sources of the data and say where to search, collect, transform and integrate data.

2) Information needs determine the design of the analytical database, the repository of data ETL process is responsible for integrating and transferring the data to achieve the objective of the final solution.

\subsection{ERP - Enterprise Resource Planning}

The origin of ERP systems dates back to the inventory control systems, later Materials Requirements Planning MRP defined in the 1970's, a concept that was later expanded by supporters of APICS in 80's to Manufacturing Resources Planning MRPII or ERP finally captured by Gartner Inc. USA in the 90's. (Chung, 2000).

ERP is a set of comprehensive and integrated applications developed by a software provider that incorporates best business practices. It is a generic development that supports a limited set of adaptations to the various companies that will use it. (Saroka, 2010). It is a "prefabricated" solution ending with incompatible information systems and inconsistent operating practices.

It is a generic solution. Its design reflects a number of assumptions about how businesses operate.

What if the particular way of operating is a strong differentiating factor or is a source of competitive advantage?

The ERP is a software package, a data quality project and finally it is a way of doing business.

\subsubsection{Features}

ERP are management information systems that integrate and automate many of the business practices associated with the operations or production aspects of a company.

ERP are integrated management systems for the enterprise. They are characterized by being composed of different parts in a single integrated application. Each part have a different use, for example, production, sales, purchasing, logistics, accounting (of various types), project management, GIS (geographic information system), inventory and warehouse control, ordering, payroll, etc. We can only define an ERP as the integration of all these parts. The opposite (a common mistake) would be considering a simple billing program as an ERP for the simple fact that a company integrates only that part. This is the fundamental difference between ERP and other management application. The ERP has everything you need for running business processes of the company. ERP cannot talk at the time that fits only one or a small part of the business processes. The very definition of ERP indicates the need for "all information available to everyone all the time."

The main objectives of ERP systems are:

- Optimization of business processes.

- Access to all information in a reliable, accurate and timely (data integrity).

- The ability to share information between all components of the organization.

- Elimination of unnecessary operations and data reengineering.

The fundamental purpose of an ERP is to provide support to business clients, fast response to their problems, and an efficient management of information to allow timely decisions and reduced overall operating costs.

\section{Results}

According to research conducted in Paraguay, have found the following results common to several companies:

- The project exceeded the original budget.

- Implementation time exceeds the estimated time, again increasing the overall cost.

- The organization demonstrates resistance to the implementation of the new system

- The system does not meet the real needs of the company.

- Lack of leadership and involvement of top management, but in the other hand, this fact was essential for success, according to companies in the research.

\section{Conclusion}

In many aspects, this research arrives at the same conclusions of Markowsky (2004), Rettig (2007) and Saroka-Collazo (2010) in Argentina, organization culture 
and resistance to change, budget exceeding, lack of leadership.

It is fundamental to avoid mistakes pointed by the research but there are still more... The idea of this research is to present a reflection paper for companies when implementing technology in their organizations, go far beyond the hardware and software implementation but go for real change, deep change, metanoia in the mind set of people and constantly learn. That's what really makes businesses smarter.

\section{REFERENCES}

[1] Collazo J. Saroka R. (2009). Informática en las Organizaciones. $1^{\circ}$ Ed. EDICON.

[2] Laudon J \& Laudon K (2005). Sistemas de Información Gerencial. $8^{\circ}$ Ed. Pearson.

[3] Rettig C. (2007). The trouble with Enterprise Software. MIT Sloan Management Review

[4] Senge P. (1990). The Fifth Discipline. Ed. Random House. 